\title{
Toxicity of pelvic nodal radiation for localized prostate cancer
}

\author{
Waqar Haque, E. Brian Butler, Bin S. Teh \\ Department of Radiation Oncology, Houston Methodist Hospital, Houston, TX, USA \\ Correspondence to: Waqar Haque, MD. Department of Radiation Oncology, Houston Methodist Hospital, Cancer Center and Research Institute, Weil \\ Cornell Medical College, Houston, TX 77030, USA. Email: waqarh786@gmail.com. \\ Provenance: This is an invited article commissioned by the Section Editor Dr. Xiao Li, MD (Department of Urology, Jiangsu Cancer Hospital \& \\ Jiangsu Institute of Cancer Research \& Nanjing Medical University Affiliated Cancer Hospital, Nanjing, China). \\ Comment on: Parry MG, Sujenthiran A, Cowling TE, et al. Treatment-Related Toxicity Using Prostate-Only Versus Prostate and Pelvic Lymph Node \\ Intensity-Modulated Radiation Therapy: A National Population-Based Study. J Clin Oncol 2019;37:1828-35.
}

Submitted Aug 27, 2019. Accepted for publication Sep 04, 2019.

doi: $10.21037 /$ atm.2019.09.57

View this article at: http://dx.doi.org/10.21037/atm.2019.09.57

External beam radiation therapy (EBRT) is a definitive treatment modality for patients with localized prostate cancer (1). The entire prostate is included within the treatment field for all patients receiving EBRT for prostate cancer. EBRT along with androgen deprivation therapy (ADT) is recommended for patients with unfavorable intermediate- or high-risk disease, with national guidelines recommending a consideration of pelvic nodal irradiation for this subset of higher risk patients (1). Multiple randomized trials have demonstrated an overall survival (OS) and local control (LC) benefit with the addition of ADT to EBRT for patients with either intermediate- or high-risk prostate cancer (2-4). However, there is a lack of data to guide management with regards to the inclusion of pelvic lymph nodes in the radiation field along with the prostate.

Three trials have specifically compared the inclusion of the pelvic lymph nodes along with the prostate in the radiation field for patients with intact prostate cancer, and none of these demonstrated a significant clinical benefit with the inclusion of the pelvic lymph nodes. Additionally, one trial has investigated this question in the post-prostatectomy setting. RTOG 77-06 compared EBRT to the prostate alone to a dose of 65 Gy to EBRT to the prostate and pelvic lymph nodes (PPLNs) to a dose of 45 Gy followed by a boost to the prostate of $20 \mathrm{~Gy}$, all in 1.8, 2.0 Gy fractions, and found no difference in LC, distant metastasis (DM), or OS between the two treatment arms (5). Toxicity results were not reported in the aforementioned trial. RTOG 9413 was a $2 \times 2$ factorial design study that sought to compare both prostate only (PO) EBRT to PPLN EBRT as well as the timing of $\mathrm{ADT}$ for patients with prostate cancer deemed to have at least a $15 \%$ risk of lymph node positive disease (6). Patients in the PO arm received 70.2 Gy to the PO, while patients in the PPLN arm received 50.4 Gy to the pelvis and prostate with a cone down boost of 19.8 Gy to the prostate, all in 1.8 Gy per fraction. No differences in outcome were observed between the PO and PPLN arms, though there was an unexpected interaction between radiation field size and timing of ADT. Importantly, acute and late toxicity were not significantly different between the treatment arms. The GETUG-01 trial compared outcomes between EBRT to the PO or EBRT to the PPLN (7). Both lowand high-risk prostate cancer patients were enrolled in this trial, though patients with high-risk disease received ADT. Patients receiving EBRT to the PO received 66-70 Gy, and patients treated to the PPLN received 46 Gy to the pelvic lymph nodes and the prostate, with a boost to 66-70 Gy to the prostate. The 10 -year OS and event free survival were similar in the two treatment arms, though a post hoc subgroup analysis suggested a benefit in pelvic radiation in lower risk patients. RTOG 05-34 was conducted on patients with prostate cancer having undergone prostatectomy who went on to develop PSA failure and compared outcomes between patients treated with either prostate fossa radiation alone, prostate fossa radiation along with ADT, and prostate fossa and pelvic lymph node radiation along with ADT (8). An improvement in biochemical control was observed in patients received EBRT to the prostate fossa, pelvic lymph nodes, and ADT when compared to patients receiving EBRT to the prostate fossa and ADT only, and 
no difference in genitourinary (GU) or gastrointestinal (GI) toxicities were reported between the three arms.

Just as there is limited data to support the benefit of inclusion of pelvic lymph nodes in the radiation field for patients with prostate cancer, there is a lack of data to determine whether or not the inclusion of pelvic lymph nodes would significantly increase toxicity. This is an especially important consideration since patients with prostate cancer tend to have a high cure rate following treatment, and would live long enough to manifest longterm toxicity due to damage to the GI or GU tracts. Small studies conducted in both the pre-intensity modulated radiation therapy (IMRT) and post-IMRT era have demonstrated worse GI side effects with the inclusion of pelvic lymph nodes in the radiation therapy field (8-11). However, other trials, also in both the pre- and postIMRT era, have shown no differences in toxicities with the inclusion of pelvic lymph nodes, though these trials were not powered to demonstrate differences in toxicities $(6,8)$.

It is in this context that investigators from the UK conducted a study using hospital based data to compare toxicities with either PO or PPLN EBRT for patients with prostate cancer (12). Using data from the English Cancer Registry Data, the National Radiotherapy Dataset (RTDS), and Hospital Episode Statistics (HES), investigators sought to compare GI and GU toxicity rates between men treated with PO or PPLN to determine if the inclusion of the pelvic lymph nodes leads to higher toxicity rates. The study was conducted in men diagnosed with prostate cancer treated with definitive EBRT between January 01, 2010 to December 10, 2013. In order to be included in the study, men had to have either high-risk or locally advanced prostate cancer and have a record of the radiation therapy dose and fractionation. The outcome was a GI or GU toxicity severe enough to require either a diagnostic or therapeutic procedure, and this was determined by evaluation of either a procedure code or diagnosis code in the patient's HES record. The authors indicated that such a procedure would signify having at least a grade 3 toxicity according to the National Cancer Institute Common Toxicity Criteria for Adverse Events (CTCAE). Patients were divided into two groups based on whether they were treated to the PO or the PPLN. Demographic and clinical characteristics were compared between the two groups using the chi-squared test. The competing risks method was used to compare the 3-year cumulative incidences of GI and $\mathrm{GU}$ toxicities, and competing risks regression according to the Fine and Gray method was used to estimate the subdistribution hazard ratio (sHRs) with $95 \%$ confidence intervals (CIs) to compare the toxicity rates between the two groups of patients.

A total of 3,845 men met the inclusion criteria, of whom 780 (20\%) received PPLN. Men receiving PPLN were more likely to be $\leq 70$ years old, have more advanced disease, and have poor socioeconomic status. The median followup time was 2.7 years. Similar toxicity rates were observed between the two groups of patients. The 3-year cumulative incidence of GI toxicity was $14 \%$ in the PPLN group and also $14 \%$ in the $\mathrm{PO}$ group. The 3 -year cumulative incidence of GU toxicity was $9 \%$ in the PPLN group and $8 \%$ in the PO group. The adjusted competing risk regression analysis demonstrated that the incidence of GI toxicity for men treated with PPLN was similar to those treated with PO RT (sHR 1.00; 95\% CI, 0.80-1.24, P=0.97); similarly, the GU toxicity for men treated with PPLN was also found to be similar to those treated with PO RT (sHR, 1.10; 95\% CI, $0.83-1.46, \mathrm{P}=0.50)$. The authors of the study concluded that the inclusion of the pelvic lymph nodes in the radiation field was not associated with an increased GI or GU toxicity at 3 years, but that greater follow-up is required to determine its impact on late GU toxicity.

This article had several strengths which suggest that its result may be applicable to patients being treated in clinical settings worldwide. First, since the study was performed in patients being treated after 2010, all patients were treated using modern radiation therapy techniques including IMRT. Since IMRT accounts for nearly $96 \%$ of all patients with prostate cancer treated with EBRT in the US, its use in the present study makes its findings generalizable to the US population (13). Second, the present study was conducted on a large sample size of patients, with a total of 3,845 patients included for analysis. The benefit of using such a large sample of patients is that this minimizes the risk of outliers confounding the outcomes and provides greater validity to the conclusions made from the statistical tests. Finally, the authors quantified the toxicity rates by using validated procedure codes to identify complications severe enough to require a procedure for management or correction of either a GI or GU toxicity. In doing so they were able to use an objective measure for treatment that is not subject to the biases of the recording physician and compare an accurate representation of the deleterious effects of EBRT that patients experienced.

The study by Parry et al. also contained significant limitations that may limit the strength of its conclusions. Perhaps most importantly, the study only had a median 
follow-up time of 2.7 years. It is possible that with longer follow-up, more long-term GI and GU toxicities may have manifested themselves in the PPLN arm and consequently, the conclusions must be tempered by the fact that this study has a limited long-term follow-up. Second, due to limitations of the data contained in the RTDS, the investigators were not able to report the dose delivered to the pelvic lymph nodes. It is possible that the dose delivered to the pelvic lymph nodes in the PPLN arm was low, and that this explains the lack of increased toxicity observed in this group of patients. Third, there was limited information regarding the radiation therapy fractionation pattern of the patients receiving radiation therapy. Recent guidelines by the American Society of Radiation Oncology and American Urological Association suggest that hypofractionation be considered in nearly all patients receiving EBRT for localized prostate cancer (14). The present study reports that the median dose delivered was 74 Gy, and the median dose per fraction was 2 Gy, but does not have data regarding the use of hypofractionation in each treatment arm. It is possible that the use of hypofractionation may impact the rates of toxicities caused by radiation therapy, and the lack of data regarding its use detracts from the generalizability of the conclusions of this study. Fourth, while the procedure codes used to determine the presence of a GI or GU toxicity were standardized across the treating institutions, they are only instituted in the case of a Grade 3 or higher toxicity. This means that the study did not account for either a Grade 1 or Grade 2 toxicity, and it is possible that there was a higher rate of Grade 1 or Grade 2 toxicities in the PPLN arm, but that this would not have been captured by the data collection methodology employed by the investigators. Additionally, the study by Parry et al. was conducted exclusively in patients treated with IMRT using photons. Previous studies have suggested that proton based therapy (PBT) may as associated with greater toxicity than treatment with photon based IMRT (13), and therefore it is possible that the toxicity profile of patients treated with PBT to the PPLN may be greater than that of patients treated with $\mathrm{PBT}$ to the PO. The present study, consisting solely of patients treated with photons, is unable to shed light on the toxicity differences associated with the volume of treatment for patients receiving treatment with PBT. Finally, there was no description of the type of daily imaging used to ensure accurate placement of patients during radiation therapy, or the type of planning target volume (PTV) expansions used on the pelvic lymph nodes. It is possible that due to daily image guided radiation therapy
(IGRT) the clinics in the current trial used smaller PTV expansions on the lymph nodes, limiting the volume treated and which may have led to the lack of observed increased toxicity associated with treatment of PPLN. However, this information is not available in the manuscript.

Overall, the study by Parry et al. is an important contribution to the literature since it showed that in a large sample size of patients with prostate cancer treated using modern radiation therapy techniques, there was no increase in GI or GU toxicity when pelvic lymph nodes are included in the radiation therapy field. We eagerly await the result of research studies that are currently ongoing (15-17) in order to determine whether there is a clinical benefit associated with the inclusion of pelvic lymph nodes in the radiation field, and which patient populations with prostate cancer may benefit most from the inclusion of pelvic lymph nodes in the treatment volume.

\section{Acknowledgments}

None.

\section{Footnote}

Conflicts of Interest: The authors have no conflicts of interest to declare.

Ethical Statement: The authors are accountable for all aspects of the work in ensuring that questions related to the accuracy or integrity of any part of the work are appropriately investigated and resolved.

\section{References}

1. National Comprehensive Cancer Network Guidelines Version 2.2019. Prostate Cancer. Accessed August 2019. Available online: http://nccn.rg/profesionals/physician_ gls/pdf/prostate.pdf

2. Roach M 3rd, Bae K, Speight J, et al. Short-term neoadjuvant androgen deprivation therapy and externalbeam radiotherapy for locally advanced prostate cancer: long- term results of RTOG 8610. J Clin Oncol 2008;26:585-91.

3. Jones CU, Hunt D, McGowan DG, et al. Radiotherapy and short-term androgen deprivation for localized prostate cancer. N Engl J Med 2011;365:107-18.

4. Pilepich MV, Winter K, Lawton CA, et al. Androgen suppression adjuvant to definitive radiotherapy in prostate 
carcinoma--long-term results of phase III RTOG 85-31. Int J Radiat Oncol Biol Phys 2005;61:1285-90.

5. Asbell SO, Krall JM, Pilepich MV, et al. Elective pelvic irradiation in Stage A2, B carcinoma of the prostate: analysis of RTOG 77-06. Int J Radiat Oncol Biol Phys 1988;15:1307-16.

6. Lawton CA, DeSilvio M, Roach M 3rd, et al. An update of the Phase III Trial comparing whole- pelvic (WP) to prostate only (PO) radiotherapy and neoadjuvant to adjuvant total androgen suppression (TAS): updated analysis of RTOG 94-13, with emphasis on unexpected hormone/ radiation interactions. Int J Radiat Oncol Biol Phys 2007;69:646-55.

7. Pommier P, Chaubad S, Lagrange JL, et al. Is there a role for pelvic irradiation in localized prostate adenocarcinoma? Update of the long-term survival results of the GETUG-01 randomized study. Int J Radiat Oncol Biol Phys 2016;96:759-69.

8. Pollack A, Karrison TG, Balogh AG Jr, et al. Short Term Androgen Deprivation Therapy Without or With Pelvic Lymph Node Treatment Added to Prostate Bed Only Salvage Radiotherapy: The NRG Oncology/RTOG 0534 SPPORT Trial. Int J Radiat Oncol Biol Phys 2018;102:1605.

9. Aizer AA, Yu JB, McKeon AM, et al. Whole pelvic radiotherapy versus prostate only radiotherapy in the management of locally advanced or aggressive prostate adenocarcinoma. Int J Radiat Oncol Biol Phys 2009;75:1344-9.

10. Pinkawa M, Piroth MD, Holy R, et al. Quality of life after whole pelvic versus prostate- only external beam

Cite this article as: Haque W, Butler EB, Teh BS. Toxicity of pelvic nodal radiation for localized prostate cancer. Ann Transl Med 2019;7(Suppl 8):S359. doi: 10.21037/atm.2019.09.57 radiotherapy for prostate cancer: a matched- pair comparison. Int J Radiat Oncol Biol Phys 2011;81:23-8.

11. Deville C, Both S, Hwang WT, et al. Clinical toxicities and dosimetric parameters after whole-pelvis versus prostateonly intensity-modulated radiation therapy for prostate cancer. Int J Radiat Oncol Biol Phys 2010;78:763-72.

12. Parry MG, Sujenthiran A, Cowling TE, et al. TreatmentRelated Toxicity Using Prostate-Only Versus Prostate and Pelvic Lymph Node Intensity-Modulated Radiation Therapy: A National Population-Based Study. J Clin Oncol 2019;37:1828-35.

13. Sheets NC, Goldin GH, Meyer AM, et al. Intensitymodulated radiation therapy, proton therapy, or conformal radiation therapy and morbidity and disease control in localized prostate cancer. JAMA 2012;307:1611-20.

14. Morgan SC, Hoffman K, Loblaw DA, et al. Hypofractionated Radiation Therapy for Localized Prostate Cancer: Executive Summary of an ASTRO, ASCO and AUA Evidence-Based Guideline. J Urol 2019;201:528-34.

15. National Institutes of Health: A Study of Prostate and Pelvis Versus Prostate Alone Treatment for Locally Advanced Prostate Cancer (PIVOTAL). Available online: http://clinicaltrials.gov/ct2/show/NCT01685190

16. SWOG Cancer Research Network: CTSU/RTOG-0924. Available online: https://www.swog.org/clinical-trials/ ctsurtog-0924

17. NHS Health Research Authority: PIVOTALBoost. Available online: http://www.hra.nhs.uk/planning-andimproving-research/application-summaries/researchsummaries/pivotalboost/ 